\title{
VAC.IO - Placental Transfer and Kinetics of Neutralizing Antibodies for Dengue Virus (DENV) in maternal-infant cohort community of Manguinhos
}

Ana Claudia Machado Duarte ${ }^{1 *}$; Stephanie Almeida ${ }^{1}$; Kátia Paulino ${ }^{1}$; Renata Pedro Saraiva ${ }^{2}$;

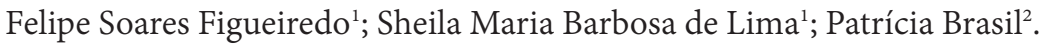

1Fiocruz/Bio-Manguinhos;

2Fiocruz/INI.

Introdução: Dengue is an important arbovirus that is currently a serious public health problem in Brazil and in the world. The Dengue virus has been circulating in the country for several decades and it has been responsible for epidemics since its reintroduction. However, the epidemiological pattern has undergone changes in the age distribution and worsening of clinical symptoms in infants, which may be associated with the presence of maternal antiDengue antibodies in neonates. Despite that, the studies including this population group are unfrequently. In this context, a prospective study has been conducted with pregnant and infants from community of Manguinhos between 2012 and 2014.

Objective: The main objective was to verify the immunological profile against Dengue virus (for all serotypes) in a maternal-infant cohort, as well as to evaluate the efficiency of maternal neutralizing antibodies transfer to the neonates. Kinetics of the neutralizing antibodies during the first year of life was also evaluated to determine the "seroreversion" time of maternal antibodies for each serotype (DENV1-4).

Methodology: Blood samples were collected from 502 pregnant woman in the last trimester of pregnancy, from 298 umbilical cord and from 298 childrens during the first year of life (at least three colection per children). We tested the samples for presence of neutralizing antibodies to all Dengue virus serotypes by Plaque Reduction Neutralization Test (PRNT).

Results: We observed that $89 \%$ of pregnant women had previous immunity to Dengue. In addition, we verified that there was an efficient transfer of maternal antibodies to all serotypes, and that maternal neutralizing antibodies seroreversion occurred between the 10th and 12th month of life. During the follow-up of the kinetics of maternal antibody decay, some infants presented increasing levels of neutralizing antibodies. For DENV-1 and DENV-2, there was an increase from the 6th month, in $31 \%$ and $20 \%$ of the following infants, respectively. For DENV-4, it was observed in $14 \%$ of the infants between 2 nd and 6th month age. For DENV-3, there was no increasing.

Conclusion: Our results indicate high transmissibility of DENV, evidencing that an expressive portion of the infants in the studied area are exposed to infection, although they still have circulating maternal antibodies. Thus, additional studies to investigate serotype-specific immunity in the pregnant population and the incidence of dengue in the first years of life are necessary. It is important to understand the dynamics of these neutralizing antibodies in children in this age group in order to define future vaccination regimen.

Keywords: Dengue virus; neutralizing antibodies; maternal-infant cohort 